\title{
SI: Organic crystal polymorphism: A benchmark for dispersion corrected mean field electronic structure methods
}

\author{
Jan Gerit Brandenburg $^{a *}$ And Stefan Grimme ${ }^{a *}$
}

${ }^{a}$ Mulliken Center for Theoretical Chemistry, Institute for Physical und Theoretical Chemistry, University of Bonn, Beringstr. 4-6, D-53115 Bonn Germany.

E-mail: gerit.brandenburg@thch.uni-bonn.de,grimme@thch.uni-bonn.de 


\section{Benchmark set POLY59}

Table 1. Summary of all systems contained in the POLY59 benchmark set. The zero indicates the experimentally found structure.

\begin{tabular}{|c|c|c|c|c|c|c|c|}
\hline no. & sym. & cell & $Z$ & no. & sym. & cell & $Z$ \\
\hline $22-0$ & $P 2_{1} / n$ & monoclinic & 4 & $24-4$ & $P 2_{1} / c$ & monoclinic & 4 \\
\hline $22-1$ & $P 2_{1} / c$ & monoclinic & 4 & $24-5$ & $P 2_{1} / c$ & monoclinic & 4 \\
\hline $22-2$ & $P 2_{1} / n$ & monoclinic & 4 & $24-6$ & $P 2_{1} 2_{1} 2_{1}$ & orthorhombic & 4 \\
\hline $22-3$ & $P 2_{1} / c$ & monoclinic & 4 & $24-7$ & $P 2_{1} / c$ & monoclinic & 4 \\
\hline $22-4$ & $P 2_{1} / n$ & monoclinic & 4 & $24-8$ & $P n a 2_{1}$ & orthorhombic & 4 \\
\hline $22-5$ & $P \overline{1}$ & triclinic & 2 & $24-9$ & $P 2_{1} 2_{1} 2_{1}$ & orthorhombic & 4 \\
\hline $22-6$ & $P \overline{1}$ & triclinic & 2 & $24-10$ & $P 2_{1} / c$ & monoclinic & 4 \\
\hline $22-7$ & $P 2_{1}$ & monoclinic & 2 & $25-0$ & $P 2_{1} / c$ & monoclinic & 4 \\
\hline $22-8$ & $P 2_{1} / n$ & monoclinic & 4 & $25-1$ & $\mathrm{Pbca}$ & orthorhombic & 8 \\
\hline $22-9$ & $\operatorname{Pna} 2_{1}$ & orthorhombic & 4 & $25-2$ & $P \overline{1}$ & triclinic & 2 \\
\hline $22-10$ & $P 2_{1} / c$ & monoclinic & 4 & $25-3$ & $P 2_{1} / c$ & monoclinic & 4 \\
\hline $23-0 \alpha$ & $P 2_{1} / c$ & monoclinic & 4 & $25-4$ & $P 2_{1} / c$ & monoclinic & 4 \\
\hline $23-0 \beta$ & $P \overline{1}$ & triclinic & 2 & $25-5$ & $P \overline{1}$ & triclinic & 2 \\
\hline $23-0 \gamma$ & $P \overline{1}$ & triclinic & 4 & $25-6$ & $P \overline{1}$ & triclinic & 2 \\
\hline $23-0 \delta$ & $P 2_{1} / n$ & monoclinic & 4 & $25-7$ & $P \overline{1}$ & triclinic & 2 \\
\hline $23-0 \epsilon$ & $P \overline{1}$ & triclinic & 4 & $25-8$ & $P \overline{1}$ & triclinic & 2 \\
\hline $23-1$ & $P \overline{1}$ & triclinic & 2 & $25-9$ & $P 2_{1} / c$ & monoclinic & 4 \\
\hline $23-2$ & $P \overline{1}$ & triclinic & 2 & $25-10$ & $P 2_{1} / c$ & monoclinic & 4 \\
\hline $23-3$ & $P \overline{1}$ & triclinic & 2 & $26-0$ & $P \overline{1}$ & triclinic & 2 \\
\hline $23-4$ & $P \overline{1}$ & triclinic & 2 & $26-1$ & $P 2_{1} / n$ & monoclinic & 4 \\
\hline $23-5$ & $P \overline{1}$ & triclinic & 2 & $26-2$ & $P 2_{1} / n$ & monoclinic & 4 \\
\hline $23-6$ & $P \overline{1}$ & triclinic & 2 & $26-3$ & $P 2_{1} / n$ & monoclinic & 4 \\
\hline $23-7$ & $P \overline{1}$ & triclinic & 2 & $26-4$ & $P 2_{1} / n$ & monoclinic & 4 \\
\hline $23-8$ & $P \overline{1}$ & triclinic & 2 & $26-5$ & $P 2_{1} / c$ & monoclinic & 4 \\
\hline $23-9$ & $P 2_{1} / c$ & monoclinic & 4 & $26-6$ & $P \overline{1}$ & triclinic & 2 \\
\hline $23-10$ & $P 2_{1} / n$ & monoclinic & 4 & $26-7$ & $C 2 / c$ & monoclinic & 8 \\
\hline $24-0$ & $P 2_{1} / c$ & monoclinic & 4 & $26-8$ & $P 2_{1} / n$ & monoclinic & 4 \\
\hline $24-1$ & $P 2_{1} / c$ & monoclinic & 4 & $26-9$ & xxx & $\mathrm{xxx}$ & 4 \\
\hline $24-2$ & $P 2_{1} / n$ & monoclinic & 4 & $26-10$ & $\mathrm{xxx}$ & $\mathrm{xxx}$ & 4 \\
\hline $24-3$ & $P 2_{1} / c$ & monoclinic & 4 & & & & \\
\hline
\end{tabular}


2. POLY59: Geometries

Table 2. Benchmark geometries of the experimentally realized blind test polymorphs isotropically

\begin{tabular}{|c|c|c|c|c|c|c|c|}
\hline & $\mathrm{a}$ & $\bar{b}$ & ${ }^{(1)} \mathrm{c}$ & $\alpha$ & $\bar{\beta}$ & ${ }^{(2)} \gamma$ & ${ }^{(3)} \mathrm{vol}$ \\
\hline & & & $22-0$ & & & & \\
\hline ref. & 11.906 & 6.673 & 12.555 & 90.0 & 108.6 & 90.0 & 945.50 \\
\hline TPSS-D3 & 11.909 & 6.646 & 12.511 & 89.9 & 108.5 & 90.0 & 938.77 \\
\hline PBE-MBD & 12.103 & 6.756 & 12.865 & 90.0 & 109.7 & 90.0 & 990.57 \\
\hline $\mathrm{HF}-3 \mathrm{c}$ & 11.449 & 6.553 & $\begin{array}{r}12.312 \\
23-0 \alpha\end{array}$ & 89.9 & 105.7 & 90.0 & 889.43 \\
\hline ref. & 10.980 & 10.356 & 15.968 & 90.0 & 95.7 & 90.0 & 1806.63 \\
\hline TPSS-D3 & 10.657 & 10.436 & 16.357 & 90.0 & 93.7 & 90.0 & 1815.23 \\
\hline PBE-MBD & 10.803 & 10.489 & 16.335 & 90.0 & 94.4 & 90.0 & 1845.55 \\
\hline $\mathrm{HF}-3 \mathrm{c}$ & 10.462 & 10.521 & $\begin{array}{r}16.342 \\
23-0 \beta\end{array}$ & 90.0 & 92.0 & 90.0 & 1797.67 \\
\hline ref. & 6.901 & 7.688 & 18.619 & 85.3 & 80.8 & 65.8 & 888.49 \\
\hline TPSS-D3 & 7.214 & 7.692 & 17.631 & 88.5 & 80.8 & 64.8 & 873.05 \\
\hline PBE-MBD & 7.221 & 7.770 & 17.943 & 87.6 & 80.5 & 64.5 & 895.57 \\
\hline $\mathrm{HF}-3 \mathrm{c}$ & 7.185 & 7.734 & $\begin{array}{r}17.317 \\
23-0 \gamma\end{array}$ & 89.5 & 80.8 & 65.4 & 862.05 \\
\hline ref. & 7.482 & 11.793 & 20.026 & 84.8 & 85.4 & 80.1 & 1729.58 \\
\hline TPSS-D3 & 7.430 & 11.995 & 19.802 & 86.4 & 89.1 & 83.0 & 1748.24 \\
\hline PBE-MBD & 7.492 & 12.011 & 19.977 & 86.1 & 88.4 & 82.9 & 1779.30 \\
\hline $\mathrm{HF}-3 \mathrm{c}$ & 7.380 & 11.860 & $\begin{array}{r}19.716 \\
23-0 \delta\end{array}$ & 87.4 & 90.4 & 83.2 & 1711.73 \\
\hline ref. & 13.648 & 10.544 & 13.837 & 90.0 & 113.6 & 90.0 & 1824.28 \\
\hline TPSS-D3 & 13.739 & 10.591 & 13.460 & 89.1 & 111.4 & 90.7 & 1823.51 \\
\hline PBE-MBD & 13.789 & 10.632 & 13.553 & 89.2 & 111.8 & 90.6 & 1845.08 \\
\hline $\mathrm{HF}-3 \mathrm{c}$ & 14.638 & 10.367 & $\begin{array}{r}13.049 \\
23-0 \epsilon\end{array}$ & 90.0 & 114.2 & 90.0 & 1806.80 \\
\hline ref. & 6.575 & 11.976 & 23.982 & 102.9 & 96.7 & 97.2 & 1805.39 \\
\hline TPSS-D3 & 6.689 & 11.539 & 23.949 & 99.2 & 98.2 & 98.0 & 1781.38 \\
\hline PBE-MBD & 6.705 & 11.652 & 23.968 & 99.0 & 98.2 & 97.7 & 1806.96 \\
\hline $\mathrm{HF}-3 \mathrm{c}$ & 6.566 & 11.341 & $\begin{array}{c}24.520 \\
24-0\end{array}$ & 100.3 & 98.0 & 98.9 & 1748.47 \\
\hline ref. & 3.979 & 21.058 & 10.008 & 90.0 & 97.8 & 90.0 & 830.90 \\
\hline TPSS-D3 & 4.024 & 20.761 & 10.001 & 90.0 & 98.4 & 90.0 & 826.65 \\
\hline PBE-MBD & 4.093 & 21.008 & 10.009 & 90.0 & 98.1 & 90.0 & 852.19 \\
\hline $\mathrm{HF}-3 \mathrm{c}$ & 4.058 & 21.016 & $\begin{array}{c}10.003 \\
25-0\end{array}$ & 90.0 & 98.3 & 90.0 & 844.29 \\
\hline ref. & 10.243 & 27.109 & 7.983 & 90.0 & 109.6 & 90.0 & 2088.51 \\
\hline TPSS-D3 & 10.376 & 27.278 & 8.104 & 90.0 & 109.8 & 90.0 & 2157.53 \\
\hline PBE-MBD & 10.346 & 27.217 & 8.050 & 90.1 & 110.1 & 90.0 & 2128.64 \\
\hline $\mathrm{HF}-3 \mathrm{c}$ & 10.318 & 27.274 & $\begin{array}{l}7.773 \\
26-0\end{array}$ & 90.0 & 109.5 & 90.0 & 2061.81 \\
\hline ref. & 10.247 & 10.866 & 13.968 & 76.8 & 73.3 & 63.5 & 1323.94 \\
\hline ofPSS-Đ3: & ${ }^{14} 109.238$ & 10.872 & 13.888 & 78.1 & 73.7 & 63.8 & 1325.12 \\
\hline PBE-MBD & 10.273 & 10.960 & 13.914 & 78.2 & 73.8 & 64.0 & 1345.81 \\
\hline $\mathrm{HF}-3 \mathrm{c}$ & 10.087 & 10.740 & 13.650 & 80.5 & 75.4 & 65.6 & 1300.43 \\
\hline
\end{tabular}

(1) cell parameter in $\AA{ }^{(2)}$ cell angle in deg, ${ }^{(3)}$ cell volume per molecule in $\AA^{3}$ 


\section{POLY59: Energies}

3.1. Target $X X I I$

Table 3. Relative lattice energies of various electronic structure methods for the crystal structure prediction blind test target 22. Energies are given in $\mathrm{kcal} / \mathrm{mol}$ and relative to the experimental polymorph 22-0.

\begin{tabular}{|c|c|c|c|c|c|c|}
\hline No. & TPSS-D3 & TPSS-D3 $^{(1)}$ & $\mathrm{HF}-3 \mathrm{c}$ & DFTB3-D3 & M06L & vdW-DF2 \\
\hline $22-1$ & 0.12 & 0.44 & -0.23 & 0.63 & 0.59 & 0.21 \\
\hline $22-2$ & 0.44 & 0.12 & -0.20 & -3.41 & 2.03 & 2.90 \\
\hline $22-3$ & 0.58 & -0.12 & 0.53 & -1.80 & 1.54 & 0.98 \\
\hline $22-4$ & 0.60 & 0.37 & -0.26 & -1.65 & 1.22 & 0.82 \\
\hline $22-5$ & 1.02 & 0.48 & -0.51 & -2.03 & 2.69 & 2.65 \\
\hline $22-6$ & 1.17 & 0.72 & 0.11 & 0.77 & 1.56 & 1.48 \\
\hline $22-7$ & 1.32 & 1.34 & 1.92 & 1.90 & 1.83 & 1.26 \\
\hline $22-8$ & 0.82 & 0.46 & -0.93 & 0.99 & 0.94 & 1.37 \\
\hline $22-9$ & 0.84 & -0.06 & 0.51 & -1.33 & 2.38 & 2.88 \\
\hline $22-10$ & 0.95 & 0.65 & 0.38 & 0.26 & 1.90 & 2.21 \\
\hline rank & 1 & 3 & 5 & 7 & 1 & 1 \\
\hline$\Delta E$ & 0.00 & -0.12 & -0.98 & -3.32 & 0.00 & 0.00 \\
\hline No. & $\mathrm{PBE}$ & PBE-D2 & PBE-TS & PBE-dDsC & PBE-D3 & PBE-MBD \\
\hline $22-1$ & 0.11 & 0.13 & 0.06 & 0.20 & 0.06 & 0.43 \\
\hline $22-2$ & 3.23 & 1.45 & 1.38 & 1.95 & 1.07 & 1.76 \\
\hline $22-3$ & 1.81 & 0.78 & 0.78 & 1.02 & 0.59 & 0.99 \\
\hline $22-4$ & 0.39 & 0.60 & 0.89 & 0.80 & 0.41 & 0.56 \\
\hline $22-5$ & 2.11 & 1.67 & 1.50 & 1.64 & 1.16 & 1.80 \\
\hline $22-6$ & 0.62 & 0.97 & 1.59 & 1.15 & 1.00 & 1.09 \\
\hline $22-7$ & -0.55 & 1.27 & 1.15 & 1.32 & 1.05 & 0.89 \\
\hline $22-8$ & 1.43 & 0.88 & 1.29 & 1.14 & 0.97 & 1.07 \\
\hline $22-9$ & 3.24 & 1.54 & 1.60 & 2.47 & 1.26 & 1.82 \\
\hline $22-10$ & 1.55 & 1.10 & 1.79 & 1.55 & 1.19 & 1.44 \\
\hline rank & 2 & 1 & 1 & 1 & 1 & 1 \\
\hline$\Delta E$ & -0.12 & 0.00 & 0.00 & 0.00 & 0.00 & 0.00 \\
\hline
\end{tabular}

(1) TPSS-D3 evaluated on HF-3c geometries

\subsection{Target XXIII}


Table 4. Relative lattice energies of various electronic structure methods for the crystal structure prediction blind test target 23. Energies are given in $\mathrm{kcal} / \mathrm{mol}$ and relative to the experimental polymorph 23-0 $\alpha$.

\begin{tabular}{|c|c|c|c|c|c|c|}
\hline No. & TPSS-D3 Tl & TPSS-D3 ${ }^{(1)}$ & $\mathrm{HF}-3 \mathrm{c}$ & DFTB3-D3 & M06L & vdW-DF2 \\
\hline $23-0 \beta$ & -0.65 & -0.65 & -1.68 & -3.50 & -0.11 & -0.17 \\
\hline $23-0 \gamma$ & 0.73 & -0.25 & -1.73 & -2.47 & 0.25 & 0.94 \\
\hline $23-0 \delta$ & 0.67 & 1.40 & 1.37 & 0.68 & 1.47 & 0.38 \\
\hline $23-0 \epsilon$ & 0.29 & 0.20 & -0.85 & -2.30 & 0.92 & 0.10 \\
\hline $23-1$ & -0.30 & -0.45 & -1.25 & -2.38 & 0.35 & 1.29 \\
\hline $23-2$ & 0.21 & 0.04 & -2.29 & -4.06 & 0.25 & 0.84 \\
\hline $23-3$ & 0.05 & -0.06 & -3.53 & -5.95 & -0.86 & -0.45 \\
\hline $23-4$ & 0.17 & 0.17 & -2.11 & -3.93 & 0.28 & 0.76 \\
\hline $23-5$ & 0.10 & -0.02 & -3.16 & -5.94 & -0.71 & -0.76 \\
\hline $23-6$ & 0.50 & 0.55 & -2.30 & -5.33 & -0.80 & 1.40 \\
\hline $23-7$ & 0.74 & 0.14 & -0.55 & -3.45 & 0.32 & -0.49 \\
\hline $23-8$ & 0.95 & 0.82 & -2.13 & -5.55 & 0.07 & 3.01 \\
\hline $23-9$ & 0.40 & 0.30 & -1.50 & -1.27 & 0.99 & 0.72 \\
\hline $23-10$ & 1.30 & 0.30 & -1.06 & -2.30 & 0.77 & 1.46 \\
\hline rank & 27 & 35 & 49 & 52 & 41 & 30 \\
\hline$\Delta E_{\alpha}$ & -0.65 & -0.65 & -3.53 & -5.95 & -0.86 & -0.76 \\
\hline$\Delta E_{\beta}$ & 0.00 & 0.00 & -1.84 & -2.45 & -0.74 & -0.60 \\
\hline$\Delta E_{\gamma}$ & -1.38 & -0.40 & -1.70 & -3.48 & -1.11 & -1.71 \\
\hline$\Delta E_{\delta}$ & -1.32 & -2.05 & -4.89 & -6.63 & -2.33 & -1.14 \\
\hline$\Delta E_{\epsilon}$ & -0.94 & -0.85 & -2.68 & -3.65 & -1.78 & -0.86 \\
\hline No. & PBE & PBE-D2 & PBE-TS & PBE-dDsC & PBE-D3 & PBE-MBD \\
\hline $23-0 \beta$ & 2.71 & -1.06 & -1.22 & -0.37 & -0.39 & -0.43 \\
\hline $23-0 \gamma$ & 3.14 & -0.23 & 0.59 & 0.24 & 0.72 & 0.13 \\
\hline $23-0 \delta$ & -0.20 & 0.71 & 1.03 & 0.95 & 0.43 & 0.05 \\
\hline $23-0 \epsilon$ & 1.03 & -0.68 & 0.31 & -0.12 & -0.05 & -0.52 \\
\hline $23-1$ & 1.73 & -0.66 & 0.06 & 0.88 & 0.03 & 0.07 \\
\hline $23-2$ & 3.69 & -0.90 & -0.07 & -5.94 & 0.23 & 0.07 \\
\hline $23-3$ & 1.99 & -1.20 & -0.71 & -1.91 & -0.19 & -0.21 \\
\hline $23-4$ & 3.32 & -0.88 & -0.10 & -0.17 & 0.14 & 0.01 \\
\hline $23-5$ & 1.89 & -1.18 & -0.78 & -1.96 & -0.25 & -0.28 \\
\hline $23-6$ & 6.13 & -0.39 & -1.05 & 1.10 & 1.04 & 0.48 \\
\hline $23-7$ & 0.36 & 0.01 & 0.65 & -0.11 & 0.24 & -0.15 \\
\hline $23-8$ & 4.87 & -0.69 & 0.61 & 1.17 & 1.25 & 0.53 \\
\hline $23-9$ & 0.81 & -0.30 & 0.84 & 1.19 & 0.37 & 0.33 \\
\hline $23-10$ & 4.11 & 0.59 & 0.10 & 1.87 & 1.33 & 0.44 \\
\hline rank & 24 & 40 & 35 & 32 & 21 & 19 \\
\hline$\Delta E_{\alpha}$ & -0.20 & -1.20 & -1.22 & -5.94 & -0.39 & -0.52 \\
\hline$\Delta E_{\beta}$ & -2.91 & -0.14 & -0.00 & -5.57 & 0.00 & -0.10 \\
\hline$\underset{\text { IUCr macr }}{\Delta E_{\gamma}}$ & $\begin{array}{c}-3.35 \\
\text { sion } 2.1 .6: 2014 / 10 / 01\end{array}$ & -0.98 & -1.81 & -6.18 & -1.11 & -0.65 \\
\hline${ }^{\mathrm{I} U}{ }_{\Delta}^{\mathrm{r}} E_{\delta}^{\text {macr }}$ & $\begin{array}{c}\text { sion 2.1.6: } \dot{x}^{2014 / 10 / 01} \\
0.00\end{array}$ & -1.91 & -2.26 & -6.98 & -0.82 & -0.57 \\
\hline$\Delta E_{\epsilon}$ & -1.23 & -0.53 & -1.53 & -5.82 & -0.35 & -0.00 \\
\hline
\end{tabular}

$\overline{\overline{(1)} \text { TPSS-D3 evaluated on HF-3c geometries }}$ 
3.3. Target $X X I V$

Table 5. Relative lattice energies of various electronic structure methods for the crystal structure prediction blind test target 24. Energies are given in $\mathrm{kcal} / \mathrm{mol}$ and relative to the experimental polymorph 24-0.

\begin{tabular}{|c|c|c|c|c|c|c|}
\hline No. & TPSS-D3 & TPSS-D3 ${ }^{(1)}$ & $\mathrm{HF}-3 \mathrm{c}$ & DFTB3-D3 & M06L & vdW-DF2 \\
\hline $24-1$ & 2.23 & 2.86 & 4.67 & 3.54 & 0.94 & 2.86 \\
\hline $24-2$ & 2.33 & 2.83 & 4.71 & 5.49 & 3.46 & 2.83 \\
\hline $24-3$ & 2.63 & 2.48 & 5.30 & 4.69 & 2.36 & 2.48 \\
\hline $24-4$ & 2.82 & 3.09 & 3.83 & 4.98 & 2.46 & 3.09 \\
\hline $24-5$ & 2.94 & 2.45 & 4.42 & 4.84 & 3.10 & 2.45 \\
\hline $24-6$ & 3.34 & 2.60 & 3.27 & 4.24 & 4.58 & 2.60 \\
\hline $24-7$ & 3.38 & 4.17 & 7.44 & 6.28 & 2.81 & 4.17 \\
\hline $24-8$ & 3.63 & 3.45 & 5.35 & 6.17 & 1.71 & 3.45 \\
\hline $24-9$ & 3.72 & 4.14 & 6.64 & 9.22 & 3.42 & 4.14 \\
\hline $24-10$ & 3.88 & 3.80 & 4.65 & 4.48 & 2.36 & 3.80 \\
\hline rank & 1 & 1 & 1 & 1 & 1 & 1 \\
\hline$\Delta E$ & 0.00 & 0.00 & 0.00 & 0.00 & 0.00 & 0.00 \\
\hline No. & PBE & PBE-D2 & PBE-TS & PBE-dDsC & PBE-D3 & PBE-MBD \\
\hline $24-1$ & -0.27 & 1.63 & 2.44 & 1.60 & 1.64 & 1.52 \\
\hline $24-2$ & -0.45 & 1.75 & 3.31 & 2.78 & 2.01 & 2.14 \\
\hline $24-3$ & -0.20 & 2.28 & 3.14 & 2.79 & 2.23 & 2.40 \\
\hline $24-4$ & 0.81 & 2.40 & 2.78 & 2.25 & 2.39 & 2.04 \\
\hline $24-5$ & 1.37 & 2.60 & 3.84 & 3.33 & 2.81 & 3.01 \\
\hline $24-6$ & 0.30 & 3.54 & 4.59 & 4.26 & 3.08 & 3.21 \\
\hline $24-7$ & -0.24 & 2.83 & 3.92 & 3.50 & 2.79 & 2.79 \\
\hline $24-8$ & 2.28 & 2.84 & 3.74 & 3.27 & 3.24 & 3.34 \\
\hline $24-9$ & -1.16 & 3.14 & 4.57 & 3.87 & 3.19 & 2.98 \\
\hline $24-10$ & 2.46 & 3.51 & 3.76 & 3.15 & 3.55 & 3.51 \\
\hline rank & 6 & 1 & 1 & 1 & 1 & 1 \\
\hline$\Delta E$ & -1.12 & 0.00 & 0.00 & 0.00 & 0.00 & 0.00 \\
\hline
\end{tabular}

(1) TPSS-D3 evaluated on HF-3c geometries

3.4. Target $X X V$

IUCr macros version 2.1.6: 2014/10/01 
Table 6. Relative lattice energies of various electronic structure methods for the crystal structure prediction blind test target 25. Energies are given in $\mathrm{kcal} / \mathrm{mol}$ and relative to the experimental polymorph 25-0.

\begin{tabular}{|c|c|c|c|c|c|c|}
\hline No. & TPSS-D3 & TPSS-D3 ${ }^{(1)}$ & $\mathrm{HF}-3 \mathrm{c}$ & DFTB3-D3 & M06L & vdW-DF2 \\
\hline $25-1$ & 0.08 & 0.10 & 0.00 & -0.99 & 1.86 & 2.13 \\
\hline $25-2$ & 0.40 & -0.51 & -0.57 & -1.58 & 0.73 & -0.40 \\
\hline $25-3$ & 0.51 & -0.45 & -1.11 & -0.31 & 1.54 & 1.57 \\
\hline $25-4$ & 0.99 & 0.37 & 2.18 & 0.67 & 3.26 & 2.89 \\
\hline $25-5$ & 0.99 & 0.12 & 0.97 & 2.60 & 3.69 & 4.60 \\
\hline $25-6$ & 1.27 & 0.48 & 0.17 & 0.55 & 2.16 & 0.68 \\
\hline $25-7$ & 1.56 & 0.49 & 0.45 & 1.97 & 1.77 & 2.50 \\
\hline $25-8$ & 1.43 & 0.69 & -0.05 & -1.56 & 0.60 & -0.02 \\
\hline $25-9$ & 1.48 & 0.55 & 1.70 & 1.34 & 2.39 & 2.76 \\
\hline $25-10$ & 1.65 & 0.84 & -0.05 & -0.12 & 0.85 & 0.43 \\
\hline rank & 1 & 3 & 5 & 6 & 1 & 3 \\
\hline$\Delta E$ & 0.00 & -0.51 & -1.11 & -1.58 & 0.00 & -0.40 \\
\hline No. & PBE & PBE-D2 & PBE-TS & PBE-dDsC & PBE-D3 & PBE-MBD \\
\hline $25-1$ & 1.84 & -1.55 & 0.28 & 1.01 & 0.39 & -0.13 \\
\hline $25-2$ & 1.85 & -1.11 & 0.79 & 0.09 & 0.32 & 0.94 \\
\hline $25-3$ & 0.75 & -0.58 & 0.78 & 0.92 & 0.59 & 0.99 \\
\hline $25-4$ & -1.00 & -0.09 & 2.63 & 2.58 & 0.57 & 1.08 \\
\hline $25-5$ & 1.84 & 0.30 & 2.48 & 3.39 & 1.58 & 1.85 \\
\hline $25-6$ & 1.00 & 0.31 & 1.31 & 1.83 & 0.94 & 1.05 \\
\hline $25-7$ & 1.74 & 0.82 & 1.95 & 2.57 & 1.64 & 2.00 \\
\hline $25-8$ & 1.74 & -0.08 & 1.09 & 0.91 & 1.15 & 1.37 \\
\hline $25-9$ & 0.00 & 0.81 & 3.63 & 3.44 & 1.45 & 2.04 \\
\hline $25-10$ & -0.46 & 0.68 & 2.82 & 1.37 & 1.16 & 1.77 \\
\hline rank & 3 & 6 & 1 & 1 & 1 & 2 \\
\hline$\Delta E$ & -1.00 & -1.55 & 0.00 & 0.00 & 0.00 & -0.13 \\
\hline
\end{tabular}

$\overline{\overline{(1)} \text { TPSS-D3 evaluated on HF-3c geometries }}$

3.5. Target XXVI 
Table 7. Relative lattice energies of various electronic structure methods for the crystal structure prediction blind test target 26. Energies are given in $\mathrm{kcal} / \mathrm{mol}$ and relative to the experimental polymorph 26-0.

\begin{tabular}{|c|c|c|c|c|c|c|}
\hline No. & TPSS-D3 & TPSS-D3 ${ }^{(1)}$ & $\mathrm{HF}-3 \mathrm{c}$ & DFTB3-D3 & M06L & $\mathrm{vdW}-\mathrm{DF} 2$ \\
\hline $26-1$ & 0.07 & 0.11 & -2.98 & -1.89 & -1.20 & 1.03 \\
\hline $26-2$ & 0.35 & 0.45 & -2.03 & -1.10 & -1.50 & -0.38 \\
\hline $26-3$ & 0.46 & 0.42 & -3.17 & -3.04 & -0.49 & 2.17 \\
\hline $26-4$ & 0.54 & 0.45 & -3.22 & -3.32 & -0.40 & 2.33 \\
\hline $26-5$ & 1.06 & 1.04 & -1.43 & -4.50 & -1.05 & 2.44 \\
\hline $26-6$ & 1.56 & 1.33 & -0.10 & 0.68 & -0.00 & 0.49 \\
\hline $26-7$ & 2.59 & 2.42 & 0.00 & 2.57 & 1.32 & 3.35 \\
\hline $26-8$ & 4.40 & -0.14 & 2.22 & 4.68 & 2.14 & 2.54 \\
\hline $26-9$ & 2.12 & 1.52 & 0.59 & -1.45 & 0.57 & 1.55 \\
\hline $26-10$ & 2.55 & 2.18 & -0.12 & -0.63 & 1.10 & 5.28 \\
\hline rank & 1 & 2 & 8 & 8 & 7 & 2 \\
\hline$\Delta E$ & 0.00 & -0.14 & -3.22 & -4.50 & -1.50 & -0.38 \\
\hline No. & PBE & PBE-D2 & PBE-TS & PBE-dDsC & PBE-D3 & PBE-MBD \\
\hline $26-1$ & 3.37 & 0.04 & -0.15 & -0.05 & 0.88 & 0.56 \\
\hline $26-2$ & 2.90 & 0.38 & -1.14 & -0.90 & 0.63 & 0.25 \\
\hline $26-3$ & 4.58 & 0.76 & -0.08 & 1.02 & 1.31 & 1.65 \\
\hline $26-4$ & 4.81 & 0.77 & 0.12 & 1.09 & 1.41 & 2.95 \\
\hline $26-5$ & 5.09 & 1.47 & 0.79 & 0.89 & 2.09 & 2.12 \\
\hline $26-6$ & 2.97 & 1.74 & 0.78 & 0.57 & 1.57 & 2.81 \\
\hline $26-7$ & $6.73-$ & 3.33 & 1.64 & 1.65 & 3.48 & 4.59 \\
\hline $26-8$ & 1.14 & 4.52 & 4.15 & 4.27 & 3.87 & 3.98 \\
\hline $26-9$ & 7.02 & 1.20 & 0.55 & 0.71 & 2.40 & 2.24 \\
\hline $26-10$ & 5.63 & 2.94 & 2.98 & 3.55 & 3.68 & 3.82 \\
\hline rank & 2 & 1 & 4 & 3 & 1 & 1 \\
\hline$\Delta E$ & 0.00 & 0.00 & -1.14 & -0.90 & 0.00 & 0.00 \\
\hline
\end{tabular}

$\overline{\overline{(1)} \text { TPSS-D3 evaluated on HF-3c geometries }}$

\section{References}

literature.bib

\section{Synopsis}

Supply a synopsis of the paper for inclusion in the Table of Contents. 